per night for the 10 dark nights per lunation (4 hours per night) and one field for the 10 grey nights per lunation (1.5-2 hours per night) and two-thirds clear weather. Although the survey could proceed with the existing semi-manual fibre positioning, it is labour-intensive, messy and time consuming. Major FLAIR upgrades to give more fibres and automatic fibre-positioning would substantially improve the efficiency of the survey, perhaps by a factor of 4. A major push for a more automatic system is under way. Various options are being studied, though a fully automated, off-telescope, pick-place fibre positioning system is the ideal solution.

By the anticipated 1998/99 start date for the FLAIR-DENIS survey, all the major photographic surveys (ER, EJ, SES, I) undertaken at the UKST should be complete. Very considerable amounts of FLAIR time may become available for the FLAIR-DENIS survey, which would constitute a new UKST survey, going on the telescope whenever the conditions are too poor for photography, as well a getting other substantial amounts of allocated time.

Parker, Q. A. 1996, Spectrum, 10, 20

Rieke, G. H., Reike, M. J., \& Paul, A. E. 1993, ApJ, 336, 752

Rix, H. W. 1993, PASP, 105, 999

\section{An Obscured Galaxy Redshift Survey with FLAIR}

\section{K. Wakamatsu}

Gifu University, Gifu 501-11, Japan.

M. Malkan

UCLA, Los Angeles, CA 90024, USA.

Q. A. Parker

AAO, Coonabarabran, NSW, Australia.

\section{H. Karoji}

NOA, Mitaka 181, Tokyo, Japan.

A problem for studies of large scale structures in nearby space $\left(c z<10,000 \mathrm{~km} \mathrm{~s}^{-1}\right)$ is the presence of the Zone of Avoidance which is so large and wide on the sky that potentially important clusters and voids remain undetected. A prime example was the Ophiuchus cluster discovered by Wakamatsu and Malkan (1981) as a heavily obscured cD cluster close to the Galactic centre region $\left(l=0 \cdot 5^{\circ}, b=+9 \cdot 5^{\circ}\right)$. It is the second brightest X-ray cluster after Perseus. A hidden galaxy survey was performed by visually searching ESO/SERC Sky Survey (R and J) copy films of the region centred at $l=355^{\circ}, b=+10^{\circ}$ finding more than 4000 galaxies in six fields. Several irregular clusters adjacent to Ophiuchus were found forming a supercluster which may be connected to the
Hercules supercluster by a wall structure parallel to the local supergalactic plane (Wakamatsu et al. 1994). In front of this supercluster, an 'Ophiuchus Void' is suggested $\left(c z=4,500 \mathrm{~km} \mathrm{~s}^{-1}\right)$. The Ophiuchus supercluster at $c z=8,500 \mathrm{~km} \mathrm{~s}^{-1}$ is similar to the Hercules supercluster $\left(c z=11,000 \mathrm{~km} \mathrm{~s}^{-1}\right)$, and extends north toward the latter supercluster.

We have used FLAIR, the fibre-spectroscopy system on the UKST (Parker 1996) to study the bridge region between the two superclusters which covers $\left(16: 00 \leq \alpha \leq 17: 20,-25^{\circ} \leq \delta \leq+2 \cdot 5^{\circ}\right)$ and a void region, 1.5 hour west of a declination zone $-30^{\circ} \leq \delta \leq-15^{\circ}$. FLAIR is well matched to the number density ( $\sim 3 /$ sq. deg.) and magnitude limit $(B \leq 17 \cdot 0)$ of the survey galaxies. The region, mostly above $b \sim 15^{\circ}$, has star densities low enough for FLAIR use without severe crowding or contamination problems. So far 1500 redshifts for obscured galaxies have been obtained. The Ophiuchus supercluster extends at least one field north towards the Hercules supercluster and is surrounded by a diffuse, extended halo $\sim 20^{\circ}=30 h^{-1} \mathrm{Mpc}$ across. A new sparse 'Libra' supercluster candidate is also detected at $c z=9000 \mathrm{~km} \mathrm{~s}^{-1}$, one field south of the southern edge of the Hercules supercluster. A wall structure is clearly suggested between this and the Ophiuchus supercluster. The proposed 'Ophiuchus-Hercules Wall' formed by a local void in front of, and another behind the Ophiuchus and Libra superclusters, may form a structure as large as the Great Wall both in apparent size $\left(>70^{\circ}\right)$ and physically $\left(100 h^{-1} \mathrm{Mpc}\right)$. These two walls cross perpendicularly near Abell 2199-the northern edge of Hercules supercluster. Any 'true' 3-D orthogonality between the OphiuchusHercules Wall and the Great Wall may be crucial for understanding $0 \cdot 1 c$ scale structure whilst this local contrast of galaxy distributions may strongly affect our estimation of the Local Group motion relative to the microwave background.

Parker, Q. A. 1996, Spectrum 10, 20

Wakamatsu, K., et al. 1994, ASP Conference Series No. 67 (San Francisco: ASP), 131

Wakamatsu, K., \& Malkan, M. 1981, PASJ, 33, 57

\section{An H I Selected Sample of Galaxies: The HI Mass Function and the Surface Brightness Distribution}

Martin Zwaan, Frank Briggs
and David Sprayberry

Kapteyn Astronomical Institute, PO Box 800, NL-9700 AV Groningen, The Netherlands. zwaan@astro.rug.nl, fbriggsastro.rug.nl, dspray@astro.rug.nl

Abstract: Results from the Arecibo H I Strip Survey, an unbiased extragalactic Hi survey, combined with optical 
and $21 \mathrm{~cm}$ follow-up observations, determine the $\mathrm{HI}$ mass function and the cosmological mass density of $\mathrm{HI}$ at the present epoch. Both are consistent with earlier estimates, computed for the population of optically selected galaxies. This consistency occurs because, although the distribution of optical central surface brightnesses among galaxies is flat, we fail to find a population of galaxies with central surface brightnesses fainter than $24 B$-mag $\operatorname{arcsec}^{-2}$, even though there is no observational selection against them.

\section{Introduction}

There have been speculations that low surface brightness (LSB) galaxies and intergalactic clouds might constitute a substantial portion of the population of nearby extragalactic objects and that they might contain comparable mass to that in normal galaxies. The LSB population would escape detection optically and would not be included in the galaxy luminosity functions that are used to compute the visible baryonic content of the local Universe (Disney 1976; McGaugh 1996). On the other hand, estimates of the $\mathrm{H}$ I mass function (HIMF) based on published observations (Briggs 1990) have seemed to indicate that there is probably not any substantial population that has been missed. Weinberg et al. (1991) and Szomoru et al (1994) have come to the same conclusion. Until recently, there were no galaxy samples that could be used to address this question empirically, since the galaxies were all first identified optically.

In this paper we present results from the Arecibo $\mathrm{H}$ I Strip Survey, an unbiased $21 \mathrm{~cm}$ survey with adequate sensitivity to detect $\mathrm{H}_{\mathrm{I}}$ of very low surface density. It is of sufficient length (approximately 15 hours of RA) and depth $\left(7400 \mathrm{~km} \mathrm{~s}^{-1}\right)$ that it should be immune to fluctuations due to the large scale structure. The total sky coverage was $\sim 65$ square degrees. In the main beam, which has a FWHM of 3.2 arcmin, the survey was capable of detecting $\mathrm{HI}_{\mathrm{I}}$ masses of $6 \times 10^{5} h^{-2} M_{\odot}$ at 7 $h^{-1} \mathrm{Mpc}$ and $1.5 \times 10^{8} h^{-2} M_{\odot}$ at the full depth of the survey. The details of the Arecibo Strip Survey are described by Sorar (1994) and Briggs (1996).

The survey yielded a total of 61 detections, of which about half could be associated with cataloged galaxies listed in the NASA Extragalactic Database (NED). About five detections with galactic latitude $|b|>10^{\circ}$, where extinction is not a problem, have no obvious counterparts on the Digitised Sky Survey (DSS). The $\mathrm{HI}$ selected galaxies generally follow the same structures as optical selected galaxies. This is consistent with (1) results from Szomoru et al (1996) who fail to detect large numbers of $\mathrm{H}$ I selected galaxies in selected void fields and (2) the finding that LSB galaxies and gas-rich dwarfs lie on structures delineated by normal, high surface brightness galaxies (Mo et al. 1994).

\section{Follow up Observations}

The follow up $21 \mathrm{~cm}$ synthesis observations on the galaxies found by this survey are essential for a number of reasons. First, Arecibo is capable of detecting galaxies as far as 6 arcmin from the centre of the beam. The positional accuracy is too poor to make unambiguous identifications with catalogued galaxies or galaxies on the DSS. Second, reliable flux measurements are necessary in order to construct an HIMF. Flux measurements from the survey data can be poor if the detected galaxy is more extended than the survey beam or if it is detected at a large distance from the centre of the beam. Finally, we have found that some signals were actually caused by pairs or a small group of galaxies. This was not always obvious from looking just at survey spectra.

We took short $(20 \mathrm{~min}) 21 \mathrm{~cm}$ line observations of 55 of the 61 detected galaxies with the VLA during the D-configuration session in May 1995. The remaining six galaxies were too close to the Sun at the time of the observations. These short observations were of sufficient sensitivity to construct $\mathrm{H}$ I maps and global profiles of 52 of the 55 potential galaxies. The signal of three systems fell below the detection limit.

Optical follow up observations were carried out on the $2.5 \mathrm{~m}$ Isaac Newton Telescope on La Palma. We have been able to make B-band images of

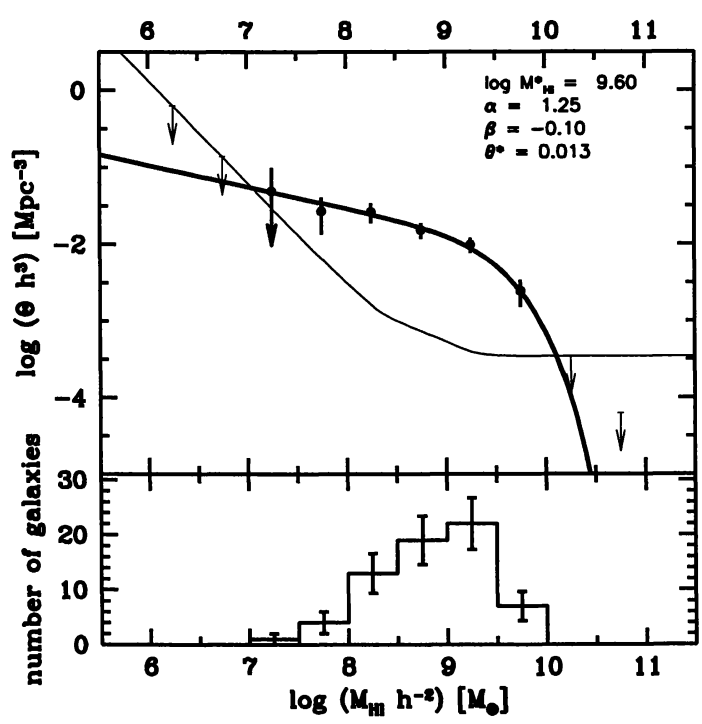

Figure 1-Lower panel: The distribution of $\mathrm{H} \mathrm{I}$ masses of the detected galaxies, with error bars given by Poisson statistics. Upper panel: The thin curve is the sensitivity of our survey. The measured $\mathrm{HI}$ mass function per half decade is shown by the points. An analytical HIMF is represented by the thick curve, using the parameters given in the upper right corner. The arrows show upper limits to the volume density of $\mathrm{HI}$ clouds. The two measurements on the right are from a complementary survey with the Arecibo telescope over the range 19,000 to $28,000 \mathrm{~km} \mathrm{~s}^{-1}$. 
24 galaxies during two observing runs in October 1995 and February 1996. Additional time has been awarded to observe the remaining galaxies. So far, we have been able to make optical identifications of all $\mathrm{HI}$ selected galaxies. No isolated $\mathrm{HI}$ clouds without stars have been found.

\section{Results}

The lower panel of Figure 1 shows the observed distribution of $\mathrm{H}$ I masses binned per half-decade, with errorbars given by Poisson statistics. The $\mathrm{H}$ I masses were calculated from either the VLA observations, or from the Arecibo measurements if the fluxes were lower than $1.0 \mathrm{Jy} \mathrm{km} \mathrm{s}{ }^{-1}$.

The inverse of the survey volume as a function of $\mathrm{HI}$ mass is indicated by the thin curve in the upper panel of Figure 1. The curve indicates the upper limit to the space density of intergalactic $\mathrm{H} \mathrm{I}$ clouds without stars as a function of $\mathrm{HI}$ mass.

The HIMF $\Theta\left(M_{\text {III }}\right)$ was determined following Schmidt's (1968) $\sum 1 / V_{\max }$ method, which consists of summing the reciprocals of the volumes corresponding to the maximum distances to which the objects could be placed and still remain within the sample. For a survey such as the Arecibo Strip Survey, $V_{\max }$ is a complicated function, dependent on velocity width, total flux, declination offset from the centre of the survey strip and feed gain, which is a function of frequency (i.e. redshift).

The solid dots in Figure 1 show the HIMF. Briggs (1990) derived an analytical expression for $\Theta$ by using a Schechter luminosity function and a relation between $\mathrm{H}_{\mathrm{I}}$ richness and optical luminosity: $L, M_{\mathrm{III}} / L \propto L^{\beta}$, where $\beta=-0 \cdot 1$. This function is represented by the thick solid curve, using $M_{\mathrm{III}} *=$ $4.0 \times 10^{9} h^{-2} M_{\odot}$, a faint end slope $\alpha=1.25$ and a normalisation $\theta^{*}=0.013$, which is a satisfactory fit to the points. The parameters of this fit agree quite well with those of optical luminosity functions. Hence, an $\mathrm{H}_{\mathrm{I}}$ selected sample of galaxies does not yield a population of gas rich dwarf galaxies (Tyson and Scalo 1988), that might be missed by optical surveys. If a large population of underluminous galaxies does exist, they must be either $\mathrm{H}$ I deficient, or have extremely low column densities $\left(N_{\mathrm{H} \mathrm{I}}<10^{18} \mathrm{~cm}^{-2}\right)$.

The cosmological mass density of $\mathrm{H}_{\mathrm{I}}$ at the present epoch, $\rho_{\mathrm{HI}}(z=0)$, can be determined from the distribution function of $\mathrm{H}_{\mathrm{I}}$ mass in galaxies. This function is plotted in Figure 2. The thick curve indicates the converted best fit $\mathrm{HI}$ mass function, while the thin curve represents the sensitivity limits. The distribution function clearly illustrates that the integral H I mass density is dominated by high mass galaxies, $M_{\text {III }} \approx 10^{9 \cdot 5} h^{-2} M_{\odot}$ which are $L^{*}$ galaxies. From this figure we derive that $\rho_{\mathrm{HI}}(z=0)=$ $4.8 \times 10^{7} h M_{\odot} \mathrm{Mpc}^{-3}$ or $3 \cdot 3 \times 10^{-33} \mathrm{~g} \mathrm{~cm}^{-3}$, with a statistical error of $25 \%$. This result agrees

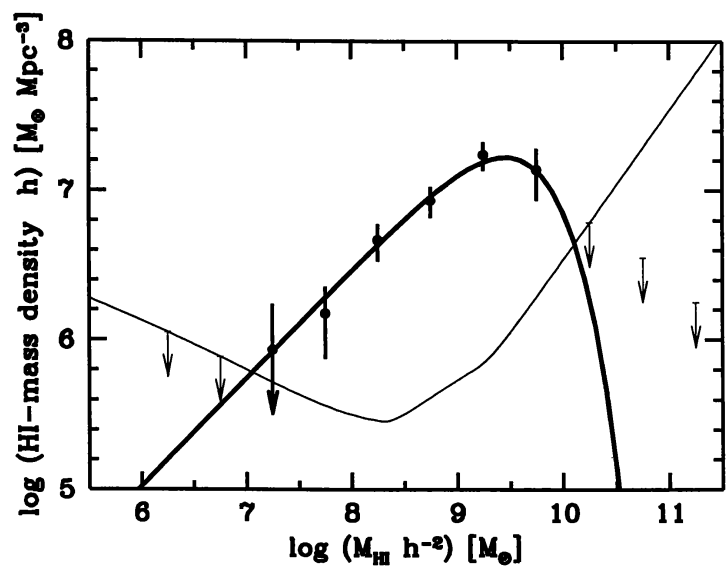

Figure 2-Space density of $\mathrm{H}$ I mass contained in objects of different masses per half decade. The thin curve indicates again the sensitivity of the survey.

surprisingly well with earlier estimates by Rao and Briggs (1993), who find the same value by using optically selected galaxies. This implies that there is not much neutral gas hidden in objects like LSB galaxies or intergalactic clouds that are missed by optical surveys. The ratio of $\mathrm{HI}$ mass density to the critical mass density of the Universe at $z=0$ is $\Omega_{\mathrm{HI}}(z=0)=(1.8 \pm 0.4) \times 10^{-4} h^{-1}$, consistent with a smooth decline of $\Omega_{\mathrm{HI}}$ from high $z$ to the present.

One final result concerns the distribution of central surface brightnesses. Since this galaxy sample is selected regardless of any optical properties, it is well suited to test the distribution function of optical surface brightnesses. The hatched area in Figure 3 indicates the possible range of values for the distribution function for the 24 galaxies observed so far. Despite the large variations due to small number statistics, it is clear that this distribution is

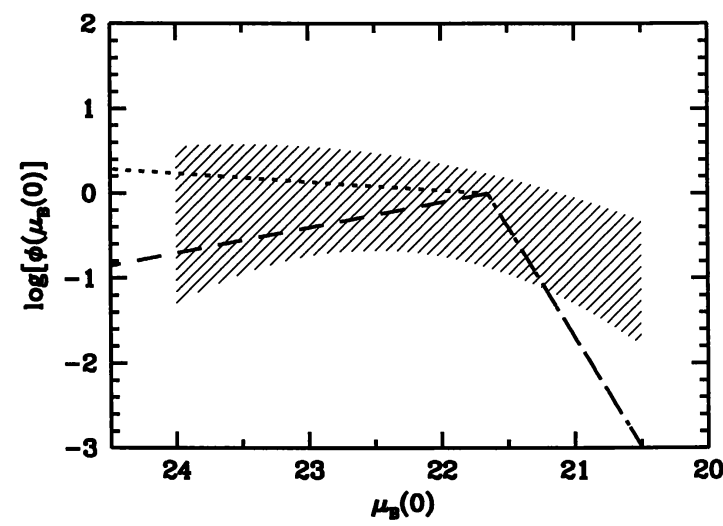

Figure 3-Volume corrected surface brightness distribution of our H I selected galaxy sample. The hatched area shows the possible range of values for this distribution function. The two lines represent the upper and lower limit to the distribution proposed by McGaugh (1996). The vertical axis is arbitrarily scaled. 
consistent with the 'flat' distribution proposed by McGaugh (1996), of which the boundaries are given by the dashed and dotted lines. It is noteworthy that no galaxies observed thus far has a central surface brightness fainter than $\sim 24.0 B$-mag $\operatorname{arcsec}^{-2}$, even though the measurement threshold is $\sim 26.5 B$ mag $\operatorname{arcsec}^{-2}$. We therefore appear to be observing a lower limit to the central surface brightness of gas-rich galaxies in the local Universe.

Briggs, F. H. 1990, AJ, 100, 999

Briggs, F. H. 1997, PASA, 14, 000
Briggs, F. H., \& Rao, S. 1993, ApJ, 417, 494

Disney, M. J. 1976, Nature, 263, 573

McGaugh, S. S. 1996, MNRAS, 280, 337

Mo, H. J., McGaugh, S. S., \& Bothun, G. D. 1994, MNRAS, 267,129

Rao, S., \& Briggs, F. 1993, ApJ, 419, 515

Schmidt, M. 1968, ApJ, 151, 393

Sorar, E. 1994, PhD Thesis, University of Pittsburgh

Szomoru, A., Guhathakurta, P., van Gorkom, J. H., Knapen, J. H., Weinberg, D. H., \& Fruchter, A. S. 1994, AJ, 108, 491

Szomoru, A., van Gorkom, J. H., Gregg, M. D., \& Strauss, M. A. 1996, AJ, 111, 2150

Tyson, N. D., \& Scalo, J. M. 1988, ApJ, 329, 618

Weinberg, D. H. 1991, ApJ, 372, L13 